\title{
Chaos in GDP
}

\author{
R. Kř́ǐz
}

\begin{abstract}
This paper presents an analysis of GDP and finds chaos in GDP. I tried to find a nonlinear lower-dimensional discrete dynamic macroeconomic model that would characterize GDP. This model is represented by a set of differential equations. I have used the Mathematica and MS Excel programs for the analysis.
\end{abstract}

Keywords: macroeconomic modeling, gross domestic product, chaos theory.

\section{Introduction}

Humanity has always been concerned with the question whether the processes in the real world are of a stochastic or deterministic nature. Answers are explored by theologians, philosophers and scientists in various fields. I take the view that real processes are more deterministic in nature. An interesting case of determinism is deterministic chaos. The only purely stochastic process is a mathematical model described by mathematical statistics. The statistical model often works and is the only possible description if we do not know the system. This also applies to economic quantities, including forecasts for GDP. In this paper I have tried to grasp the hidden essence of the problem in order to formulate a prediction more easily. The basic question is therefore the existence of chaotic behavior. If the system behaves chaotically, we are forced to accept only limited predictions. In this paper I will try to show the chaotic behavior of GDP and then propose a simple lower-dimensional system under which the system evolves.

\section{Methodology for the analysis}

I will briefly state the basic definitions and describe the basic methods for examining the input data.

\subsection{Gross domestic product}

Gross domestic product (GDP) is a major macroeconomic indicator. It measures the overall production performance of the economy. It is the total market value of all final goods and services produced within a country during some period, usually one year, expressed in monetary units [8]. It is often considered an indicator of a country's standard of living. GDP can be determined in three ways, all of which should, in principle, give the same result. They are the product (or output) approach, the in- come approach, and the expenditure approach.

$$
Y=C+I+G+(X-M)
$$

- $C$ (consumption) is normally the largest GDP component in the economy, consisting of private (household final consumption expenditure) in the economy.

- I (investment) includes business investment in equipment, for example, and does not include exchanges of existing assets.

- $G$ (government spending) is the sum of government expenditures on final goods and services.

- $X-M$ (net exports) represents gross exports $X$ - gross imports.

\subsection{Hurst exponent}

The Hurst exponent is widely used to characterize some processes. The Hurst exponent is a measure that has been widely used to evaluate the selfsimilarity and correlation properties of fractional Brownian noise, the time-series produced by a fractional (fractal) Gaussian process.

As originally defined by Mandelbrot [5], the Hurst exponent $\mathrm{H}$ describes (among other things) the scaling of the variance of a stochastic process $y(t)$,

$$
\sigma^{2}=\int_{-\infty}^{+\infty} y^{2} f(y, t) \mathrm{d} y=c t^{2 H}
$$

where $c$ is constant.

The Hurst exponent is used to evaluate the presence or absence of long-range dependence and its degree in a time-series.

The Hurst exponent $(H)$ is defined in terms of the asymptotic behavior of the rescaled range as a function of the time span of a time series, as follows

$$
E\left[\frac{R(n)}{S(n)}\right]=C n^{H} \quad \text { as } \quad n \rightarrow \infty,
$$


where $[R(n) / S(n)]$ is the rescaled range; $E[y]$ is expected value; $n$ is number of data points in a time series, $C$ is a constant.

An algorithm for calculation is used from Wikipedia [12]. To calculate the Hurst exponent, one must estimate the dependence of the rescaled range on the time span $n$ of observation. The average rescaled range is then calculated for each value of $n$. For a (partial) time series of length $\mathrm{n}, Y=$ $Y_{1}, Y_{2}, \ldots, Y_{n}$, the rescaled range is calculated as follows:

1. Create a mean-adjusted series

$$
U_{t}=Y_{t}-\frac{1}{n} \sum_{i=1}^{n} Y_{i} \quad \text { for } \quad t=1,2, \ldots, n
$$

2. Calculate the cumulative deviate series $V$;

$$
V_{t}=\sum_{i=1}^{n} U_{i} \quad \text { for } \quad t=1,2, \ldots, n
$$

3. Compute the range $R$;

$$
R(n)=\max \left(V_{1}, V_{2}, \ldots, V_{n}\right)-\min \left(V_{1}, V_{2}, \ldots, V_{n}\right)
$$

4. Compute the standard deviation $S$

$$
S(n)=\sqrt{\frac{1}{n} \sum_{i=1}^{n}\left(Y_{i}-\bar{Y}\right)^{2}}
$$

5. Calculate the rescaled range and average over all the partial time series of length $n$. The Hurst exponent is estimated by fitting the power law, according to the definition.

The values of the Hurst exponent vary between 0 and 1 , with higher values indicating a smoother trend, less volatility, and less roughness. Random walk has a Hurst exponent of 0.5.

\subsection{Fractal}

The term "fractal" was first introduced by Mandelbrot [4]. A fractal is a complicated geometric figure that, unlike a conventional complicated figure, does not simplify when it is magnified. In the way that Euclidean geometry has served as a descriptive language for classical mechanics of motion, fractal geometry is being used for the patterns produced by chaos [9].

The fractal dimension, $D$, is a statistical quantity that gives an indication of how completely a fractal appears to fill space, as one zooms down to finer and finer scales. There are many specific definitions of fractal dimension.

Hurst exponent $H$ is directly related to fractal dimension $D$, because the maximum fractal dimension for a planar tracing is 2 :

$$
D+H=2
$$

\subsection{Correlation dimension}

Correlation dimension $\left(D_{C}\right)$ describes the dimensionality of the underlying process in relation to its geometrical reconstruction in phase space. The correlation dimension is calculated using the fundamental definition. Define the correlation integral for set of data $M$ :

$$
C(r)=\frac{1}{M(M-1)} \sum_{\substack{i, j=1 \\ i \neq j}}^{M} H\left(r-\left\|y_{i}-y_{j}\right\|\right)
$$

where $H$ is the Heaviside step function.

$$
H(x)=\left\{\begin{array}{cc}
0 & y<0 \\
\frac{1}{2} & y=0 \\
1 & y>0
\end{array}\right.
$$

A Euclidean metric is used for all calculations in this paper. When a lower limit exists, the correlation dimension is then defined as

$$
\begin{aligned}
D_{C}= & \lim _{r \rightarrow 0} \frac{\ln (C(r))}{\ln (r)} \\
& M \rightarrow \infty
\end{aligned}
$$

\subsection{Lyapunov exponents}

The Lyapunov exponent or the Lyapunov characteristic exponent of a dynamical system is a quantity that characterizes the rate of separation of infinitesimally close trajectories. Quantitatively, two trajectories in phase space with initial separation $\delta Z_{0}$ diverge (provided that the divergence can be treated within the linearized approximation)

$$
\delta Z(t) \approx e^{\lambda t}\left|\delta Z_{0}\right|
$$

where $\lambda$ is the Lyapunov exponent.

The maximal Lyapunov exponent can be defined as follows:

$$
\lambda=\lim _{\substack{\delta Z_{0} \rightarrow 0 \\ t \rightarrow \infty}} \frac{1}{t} \ln \frac{|\delta Z(t)|}{\left|\delta Z_{0}\right|}
$$

The limit $\delta Z_{0} \rightarrow 0$ ensures the validity of the linear approximation at any time.

Maximal Lyapunov exponent determines a notion of predictability for a dynamical system. A positive Maximal Lyapunov exponent is usually taken as an indication that the system is chaotic (provided some 
other conditions are met, e.g., phase space compactness) [13].

\section{Introduction to nonlinear dynamics}

If the world is not linear (and there is no qualitative reason to assume that it is linear), it should be natural to model dynamical economic phenomena nonlinearly [2]. If it is present in the general system of nonlinear dynamics, a deterministic system can generate random-looking results, but may include hidden order. In this paper I have studied only a lowdimensional discrete dynamical system.

\subsection{Discrete dynamical system}

Definition from Goldsmith [6]: A discrete-time dynamical system on a set $Y$ is just the function

$$
\Phi: Y \rightarrow Y
$$

This function, often called a map, may describe the deterministic evolution of some physical system: if the system is in state $\mathrm{y}$ at time $\mathrm{t}$, then it will be in state $\Phi(y)$ at time $t+1$. The study of discrete-time dynamical systems is concerned with iterates of the map: the sequence

$$
y, \Phi(y), \Phi^{2}(y), \ldots
$$

is called the trajectory of y and the set of its points is called the orbit of $y$. These two terms are often used interchangeably, although we will remain consistent in their usage. The set $Y$ in which the states of the system exist is referred to as the phase space or state space. We will restrict our attention to maps $\Phi(y)$ such that $Y$ is a subset of $R^{d}$.

\subsection{One-dimensional discrete dynamical system}

The one-dimensional discrete system is

$$
y_{t+1}=f\left(y_{t}\right) \quad y_{t} \in \Re
$$

One-dimensional, discrete dynamical systems in economics are surely suited for demonstrating the relative easy with which complex behavior can be modeled. The mathematical properties of onedimensional dynamical systems seem to be better understood than higher-dimensional systems [2].

The logistic equation is a typical example of easy one-dimensional discrete dynamical systems which can be chaotic.

$$
y_{t+1}=\mu y_{t}\left(1-y_{t}\right)
$$

The logistic equation has been described in many books and papers e.g. [1,2,6,9,10]. The logistic equation is chaotic, when control parameter $\mu$ is between $3.5699 \ldots$ and 4 .

\section{Analysis of GDP}

\subsection{Input data}

The gross domestic product by type of expenditure in current prices is used in this paper. I have used data (quarterly, without seasonal adjustment) from the Czech Statistical Office. I analyze data from the Czech Republic between 1995 and 2010 [11].

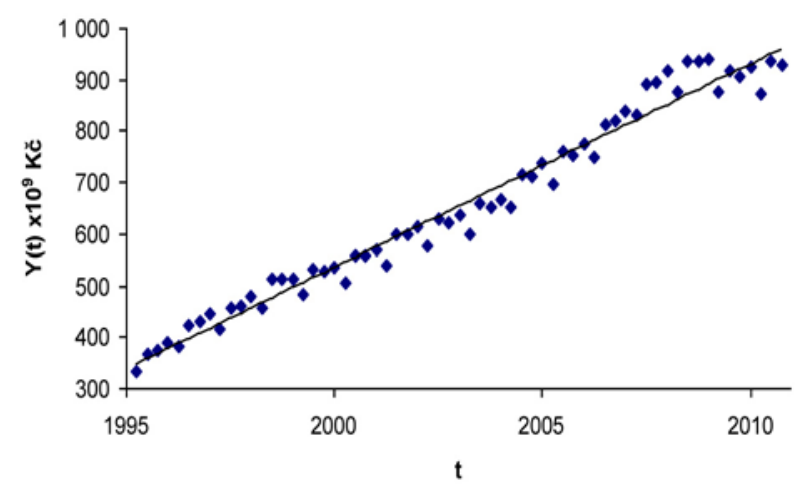

Fig. 1: GDP with linear trend of GDP

\subsection{Calculation of $H$ and $D_{c}$}

The main problem in analyzing GDP is the lack of data. Therefore, all results are only estimates.

I have computed the Hurst exponent for GDP $H=0.75$ according to the algorithm in chapter 2.2. This value is in accordance with expectations. We know that the value of $H$ is between 0 and 1 , whilst real time series are usually higher than 0.5 . If the exponent value is close to 0 or 1 , it means that the time-series has long-range dependence. Value 0.75 is directly between the stochastic and deterministic process. I think that 0.75 value is a sufficient value for credible prediction. Now we also know the fractal dimension $2-0.75=1.25$.

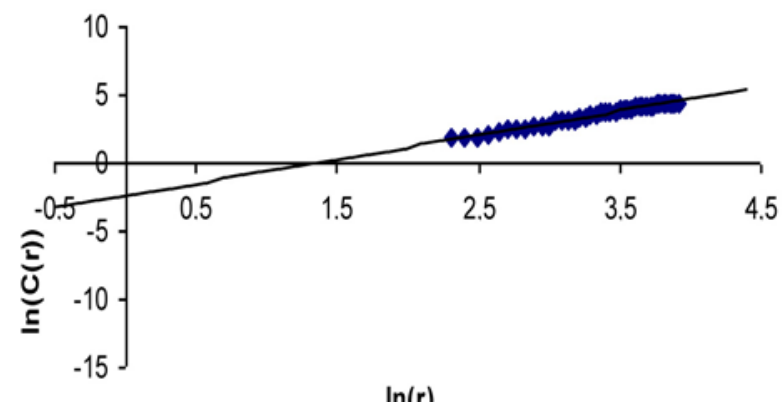

Fig. 2: Correlation integral, value with a linear trend 
The correlation dimension is calculated using the fundamental definition in Section 2.4. We have had a problem with lack of data for this computing. I have put the calculated data into a graph in logarithmic coordinates, and I have made a linear interpolation. (cf. Figure 2). On this basis, the correlation dimension for the small value of $r$ can be estimated.

The estimate of the correlation dimension is a value lower than 2 . If the correlation dimension is low, the Lyapunov exponent is positive and the Kolmogorov entropy has a finite positive value, chaos is probably present. From estimates $H$ and $D_{c}$ it can be concluded that GDP is a deterministic chaos.

\subsection{Analyzing in phase space}

In the previous section (Section 4.2) we verified the presence of chaos in GDP.

Data with a trend can cause problems for future analysis. The trend is removed by subtracting the linear interpolation. Denote GDP without trend as $Y(t)$.

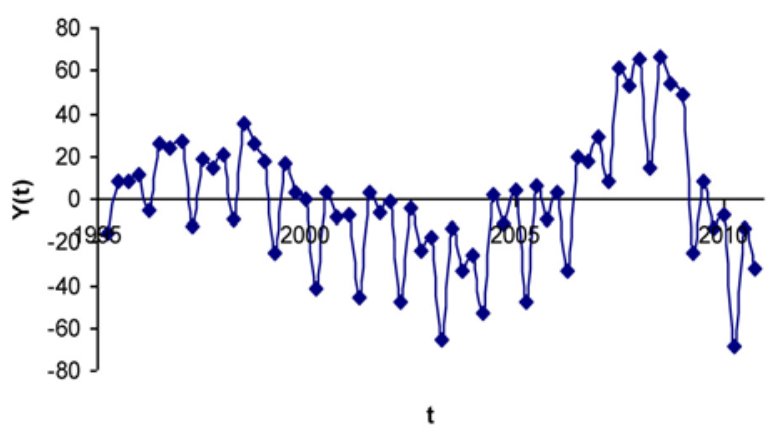

Fig. 3: GDP without trend

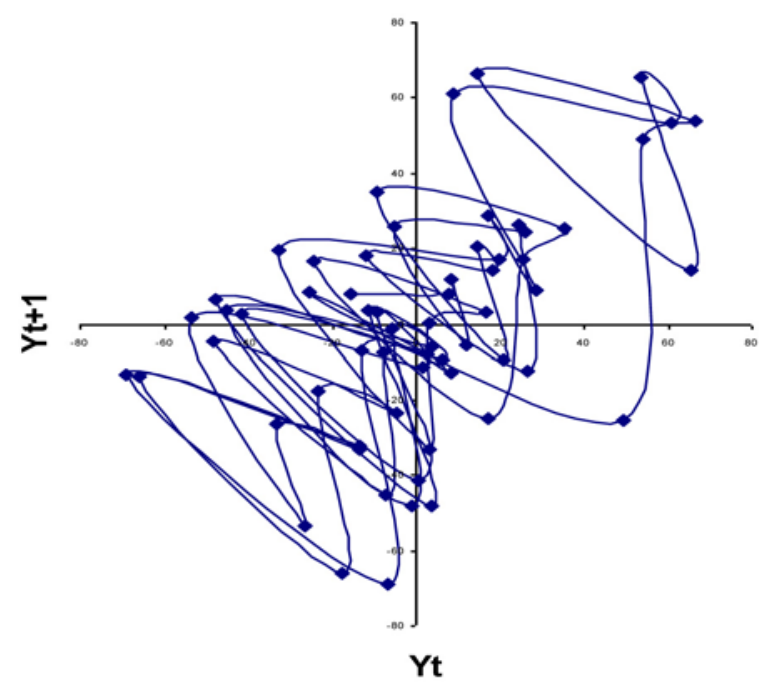

Fig. 4: GDP in phase space

A phase portrait $2 \mathrm{D}$ of GDP is constructed so that each ordered pair of $\left\{Y_{t} ; Y_{t-1}, t=2, \ldots, N\right\}$ is displayed in the plane where the $x$-axis represents the values of $Y_{t}$ and $y$-axis value $Y_{t-1}$ (cf. Figure 4$)$. The individual points $\left\{Y_{t} ; Y_{t-1}\right\}$ of phase space are connected by a smooth curve. This curve looks like a chaotic attractor. The points are located mainly in the first and third quadrant.

The phase portrait 3D of GDP is constructed so that each ordered trio of $\left\{Y_{t} ; Y_{t-1}, Y_{t-2}, t=\right.$ $3, \ldots, N\}$ is displayed in the space. The individual points $\left\{Y_{t} ; Y_{t-1}, Y_{t-2}\right\}$ of $3 \mathrm{D}$ phase space are connected by a line (cf. Figure 5 ) or covered by a surface (cf. Figure 6). Figure 6 looks very strange.

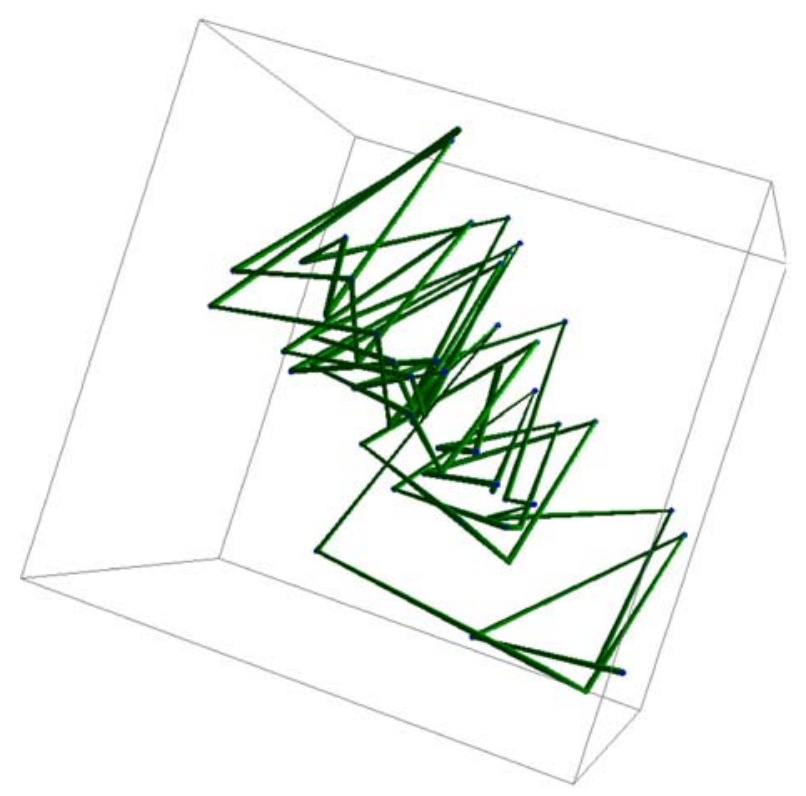

Fig. 5: GDP in 3D phase space $\left(Y_{t}, Y_{t-1}, Y_{t-2}\right)$

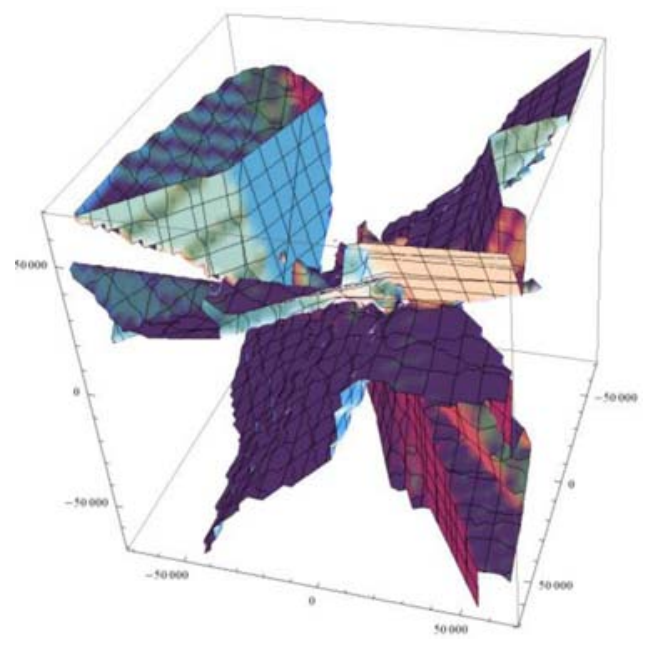

Fig. 6: GDP in 3D phase space $\left(Y_{t}, Y_{t-1}, Y_{t-2}\right)$

\section{GDP as a dynamical system}

I have tried to find a system that will be similar to GDP, based on the previous analysis. 


\subsection{Comparison of GDP with an one-dimensional discrete dynamical system}

Chaotic course $Y_{t}$ can be immediately simulated by a logistic equation, but a progression in the phase space is completely different. A logistic equation can be appropriate for simulation, but not for forecasting. If we consider a function, it should be an odd function.

I have studied several one-dimensional systems with a cubic function. It is reasonable to assume that that function has one root 0 . The desired equation can be written in the form:

$$
y_{t+1}=y_{t}\left(\alpha y_{t}^{2}-\beta\right)
$$

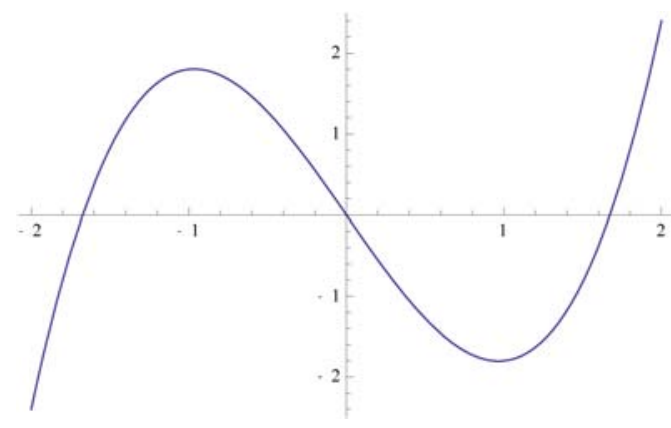

Fig. 7: Cubic function: Plot $\left[y^{\wedge} 3-2.8 y, y,-2,2\right]$

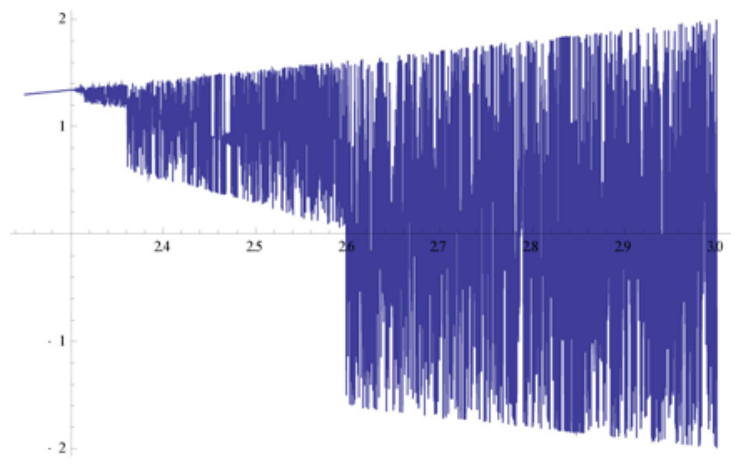

Fig. 8: Bifurcation diagram of function $y^{3}-b y,\{b, 2.25,3\}$

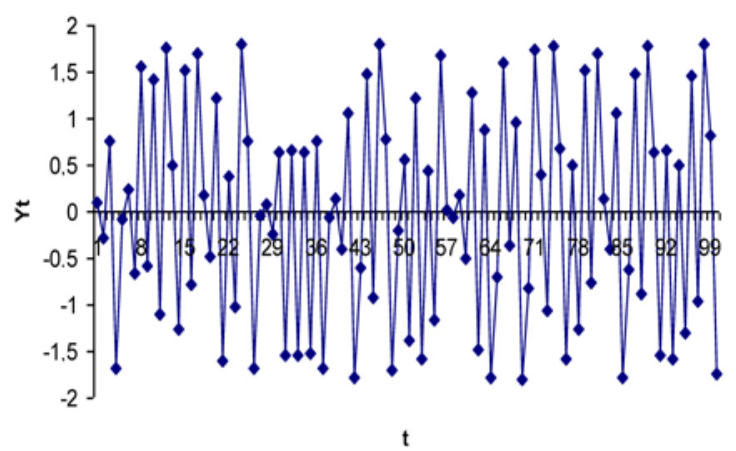

Fig. 9: Chaos in cubic function $Y_{t+1}=Y_{t}^{3}-2.8 Y_{t}$

\section{Conclusion}

I have shown in this paper that GDP can be chaotic. I found a very simple nonlinear differential equation which properly captures GDP. According to the phase portrait, it seems that the function should be odd. The most appropriate function is the cubic equation with a single zero root. It is a simple system that is easy to interpret. It should be noted that this system is not perfect and it would be useful to find a set of differential equations of higher order. The biggest problem in finding a suitable system is the lack of data. This paper analyzes GDP as such. It would be more sophisticated to create a theoretical model and calibrate it.

\section{Acknowledgement}

The research presented in the paper was supervised by doc. Ing. Helena Fialová, CSc., FEE CTU in Prague and supported by the Czech Grant Agency under grant No. 402/09/H045 "Nonlinear Dynamics in Monetary and Financial Economics. Theory and Empirical Models."

\section{References}

[1] Allen, R. G. D.: Matematická ekonomie. Academia, 1971.

[2] Lorenz, H.-W.: Nonlinear Dynamical Economics and Chaotic Motion. Springer-Verlag, 1989.

[3] Flaschel, P., Franke, R., Demmler, W.: Dynamic Macroeconomics. The MIT Press, 1997.

[4] Mandelbrot, B. B.: The Fractal Geometry of $\mathrm{Na}$ ture. W. H. Freeman and Co., 1983.

[5] Mandelbrot, B. B., Ness Van, J. W.: Fractional Brownian motions, fractional noises and applications. SIAM Rev. 10 (1968), pp. 422.

[6] Goldsmith, M.: The Maximal Lyapunov Exponent of a Time Series. Thesis, 2009.

[7] Grassberg, P., Procaccia, I.: Characterization of strange attractors, Phys. Rev. Lett. 50 (1983) 346.

[8] Fialová, H., Fiala, J.: Ekonomický slovnik. A plus, 2009.

[9] Alligood, T. K., Sauer, D. T., Yorke, J. A.: CHAOS an introduction to dynamical systems. Springer, 2000. 
[10] Chiarella, C.: The Elements of a Nonlinear Theory of Economic Dynamics. Springer-Verlag, 1990.

[11] http://www.czso.cz/eng/redakce.nsf/i/time_series

[12] http://en.wikipedia.org/wiki/Hurst_exponent

[13] http://en.wikipedia.org/wiki/Lyapunov_exponent

\section{About the author}

Radko Kříž, MSc. was born in Broumov. He graduated at the Faculty of Electrical Engineering of the Czech Technical University in Prague, specializing in
Economics and Management in Electrical Engineering, and he is currently a $\mathrm{PhD}$ student at the Faculty of Electrical Engineering of the Czech Technical University in Prague. His major fields of specialization are Macroeconomics, Energetics, Nonlinear Dynamics in Economics and Chaos Theory.

Radko Kříž

E-mail: krizradk@fel.cvut.cz

Dept. of Economics

Management and Humanities

Faculty of Electrical Engineering

Czech Technical University

Technická 2, 16627 Praha, Czech Republic 